\title{
Atlas historique et technique de la pierre à bâtir bourguignonne. Plateforme numérique mutualiste sur la nature et l'usage de la pierre
}

\section{Stéphane Büttner}

\section{(2) OpenEdition}

1 Journals

\section{Édition électronique}

URL : https://journals.openedition.org/cem/12021

DOI : 10.4000/cem. 12021

ISSN : 1954-3093

Éditeur

Centre d'études médiévales Saint-Germain d'Auxerre

Édition imprimée

Pagination : 351-355

ISSN : 1623-5770

Référence électronique

Stéphane Büttner, «Atlas historique et technique de la pierre à bâtir bourguignonne. Plateforme numérique mutualiste sur la nature et l'usage de la pierre », Bulletin du centre d'études médiévales d'Auxerre | BUCEMA [En ligne], 15 | 2011, mis en ligne le 24 août 2011, consulté le 22 septembre 2022. URL : http://journals.openedition.org/cem/12021; DOI : https://doi.org/10.4000/cem.12021

Ce document a été généré automatiquement le 22 septembre 2022.

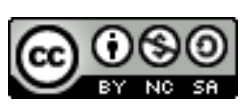

Creative Commons - Attribution - Pas d'Utilisation Commerciale - Partage dans les Mêmes Conditions 4.0 International - CC BY-NC-SA 4.0

https://creativecommons.org/licenses/by-nc-sa/4.0/ 


\title{
Atlas historique et technique de la pierre à bâtir bourguignonne. Plateforme numérique mutualiste sur la nature et l'usage de la pierre
}

\author{
Stéphane Büttner
}

1 L'objectif de cet atlas est de déterminer les arguments environnementaux qui ont défini la Bourgogne comme une région novatrice et influente dans le domaine de l'histoire de l'art et de l'architecture. La Bourgogne possède une identité fortement marquée par les arts de la construction et de la statuaire. Les ressources géologiques exploitables sont nombreuses et variées. Ces aspects font l'objet d'attentions particulières et complémentaires, tant de la part des chercheurs (archéologues, historiens, géologues) que de celles des professionnels de la filière « pierre » et de la gestion du patrimoine. La prise en compte de cet intérêt commun est l'un des points forts du projet.

2 Actuellement en cours de démarrage, ce programme est cofinancé par la Région Bourgogne (CPER) et l'Union Européenne (FEDER) avec pour principaux acteurs : David Dessandier, Marion Foucher, Jean-Pierre Garcia, Lise Leroux, Christophe Petit, Laure Saligny, Christian Sapin et Jérôme Thomas ${ }^{1}$. De manière pratique, il s'agit d'élaborer un inventaire informatisé de l'usage de la pierre dans la construction historique de la Bourgogne - Antiquité, Moyen Âge et époque moderne -, ainsi que dans la production mobilière lithique (statuaire, sarcophages, etc.). À terme, ces informations seront exploitées afin d'analyser la circulation et la diffusion des matériaux dans l'espace et dans le temps. Les résultats seront mis à disposition de la communauté scientifique, des professionnels de la restauration, de la filière « pierre » et du public, par le biais d'une publication et d'une plateforme web. 


\section{Les enjeux scientifiques et patrimoniaux}

3 Pour les historiens du bâti, la compilation et l'analyse des données permettra d'appréhender à travers les caractéristiques naturelles et anthropiques des matériaux, perçus dans leurs contextes d'utilisations, la nature même des liens entre la construction et son environnement naturel. Il s'agit de mieux saisir les éventuelles contraintes architecturales que les matériaux peuvent imposer ou subir et qui vont donc avoir une influence sur les modèles architecturaux. C'est là un élément de compréhension crucial du fonctionnement technique et économique de la construction passée. Les historiens de l'art pourront aussi y trouver matière à réflexion quant à la diffusion des modèles, aux filiations stylistiques, qu'il s'agisse d'architecture, de statuaire ou de toute autre production mobilière lithique.

4 L'outil ainsi élaboré doit permettre d'identifier les principaux bassins de production en matériaux, leurs aires de diffusion (matériaux et techniques) et d'estimer les éventuels liens entre le choix spécifique d'un matériau, le traitement technique subi et son emploi en tant que composant du bâti. Les données textuelles et archéologiques fixeront le cadre chronologique.

5 Par ce biais, l'objectif est également de valoriser la production des professionnels actuels de la pierre à travers les usages historiques et techniques des matériaux dans la région et par-delà ses frontières.

\section{Les acquis}

Plusieurs projets de bases de données sur ces thèmes ont déjà vu le jour de manière parallèle et concomitante, accumulant les informations sur les matériaux en œuvre dans la construction bourguignonne historique, ainsi que sur les sites d'extractions passés et présents. 


\section{La base « Archéomatériaux » (UMR 5594 Artehis)}

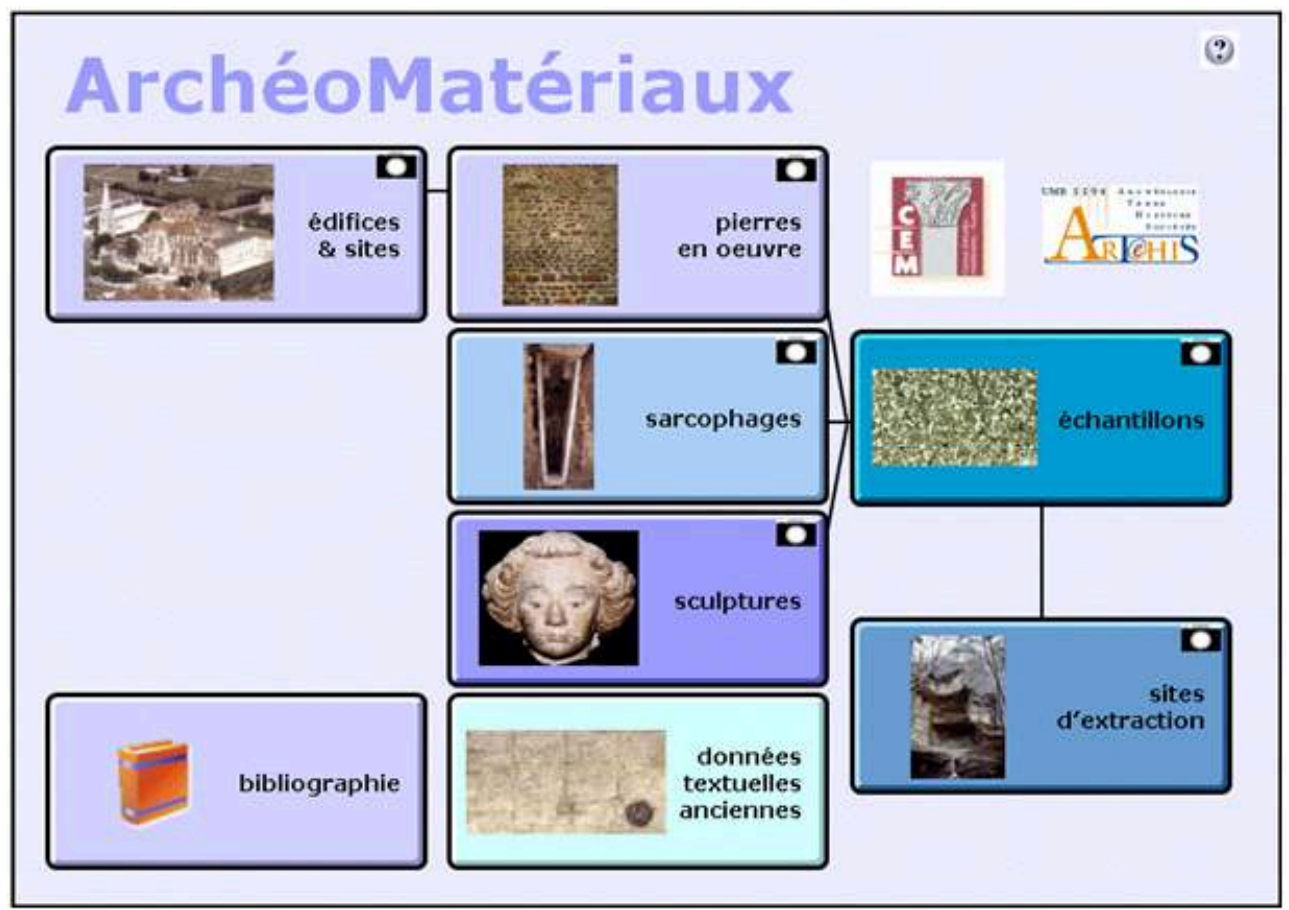

Fig. 1 - Page d'accueil de la base «Archéomatériaux».

7 Dans le cadre d'un post-doctorat $\mathrm{CNRS}^{2}$, un premier outil a été élaboré afin de raisonner sur les bases du projet et sa réalisation. Son architecture a été pensée comme structure possible d'une future plateforme mutualiste. La base "Archéomatériaux " permet ainsi d'inventorier les matériaux archéologiques d'origine géologique dans l'objectif de saisir l'histoire de l'exploitation des ressources minérales de Bourgogne et son impact sur la culture matérielle passée (fig. 1). Elle contient plusieurs modules, dont le principal concerne les matériaux utilisés dans la construction bourguignonne ancienne. Il s'agit alors de croiser les données analytiques issues de l'étude des matériaux reconnus en œuvre, donc en lien avec l'architecture, et leur datation selon des arguments archéologiques ou historiques. D'autres modules sur des objets spécifiques, comme les sarcophages ou la sculpture, ont été également développés et le champ reste ouvert. Un catalogue des données textuelles anciennes concernant les matériaux a également été associé. Cette base de données est actuellement alimentée par certains travaux de recherche menés dans le cadre de l'UMR et vise à se développer plus encore ${ }^{3}$.

\section{Les bases de données «partenaires »}

- La base « Transgéol » (Sciences Terre UB)

Le département des Sciences de la Terre de l'université de Bourgogne possède des collections de références importantes et une base de données est actuellement en cours d'élaboration afin d'en gérer l'intégralité. La prise en compte de ce corpus permettra d'avoir une partie du référentiel sur le naturel nécessaire au fonctionnement de la base de données " Archéomatériaux ».

- La base « PierBourgogne » (BRGM) 
En partenariat avec le DRIRE Bourgogne et l'association Pierre de Bourgogne, le BRGM a développé un "Système d'information sur les pierres ornementales de Bourgogne, leurs carrières d'origine et les zones potentielles d'extraction ». Sa finalité est de fournir un outil de gestion patrimoniale des pierres, notamment en fournissant des éléments d'orientation à destination des exploitants carriers et de l'administration en vue de la définition "d'espaces carrières", zones géographiques renfermant une ressource en pierre ornementale à préserver. Cette base de données aboutie est accessible sur internet ${ }^{4}$. L'intégration de ces données à la plateforme mutualiste constituera un référentiel incomparable concernant les niveaux naturels et les sites d'extraction « carrières ».

- La base « Pierre » du LRMH

Le pôle « Pierre » du laboratoire de recherche des Monuments historiques (ministère de la Culture) a, pour partie, la mission d'identifier et de rechercher la provenance des pierres des monuments historiques. Ce laboratoire possède une lithothèque et une documentation de référence en ce qui concerne les carrières et les édifices de la région Bourgogne. Ce thésaurus constituera pour le projet un centre de ressource primordial.

- Un développement international possible : la base « Limestone Sculpture Provenance Project »

11 Des collaborations sont envisagées avec le Limestone Sculpture Provenance Project, initié par le Metropolitan Museum de New York. Ce projet, qui implique plusieurs universités américaines et des chercheurs français, entreprend d'établir des correspondances entre les collections américaines et des références françaises par le biais de l'activation neutronique. Une base recensant les premiers résultats a d'ores et déjà été mise en ligne et intègre des édifices appartenant au corpus mis en œuvre dans le cadre du projet "Archéomatériaux " ${ }^{5}$. À terme, il est envisagé d'intégrer ces données dans la plateforme numérique commune.

\section{Les outils de l'expertise}

12 L'objectif est donc le développement d'une nouvelle plateforme numérique passerelle, qui serait, de fait, un outil d'expertise pluridisciplinaire exceptionnel (fig. 2). 


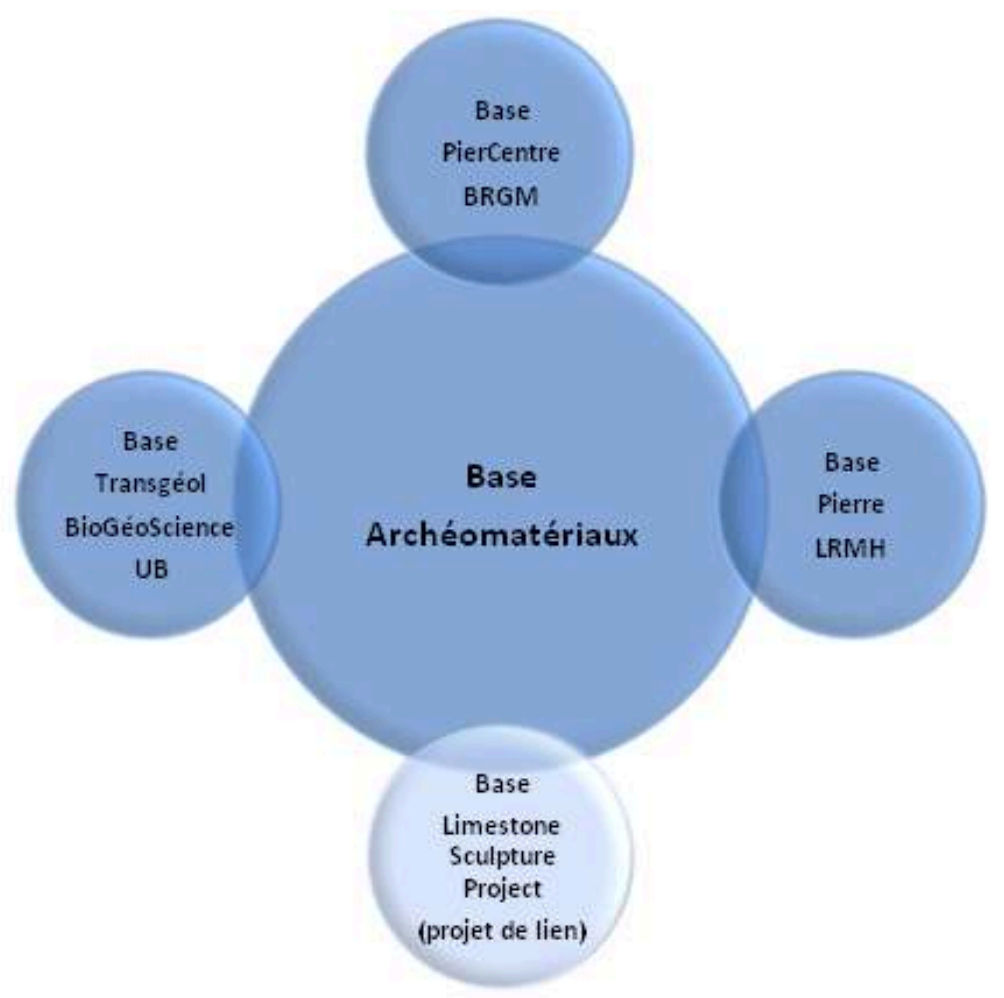

Fig. 2 - Schéma de la plateforme numérique mutualiste. partenaires, mais également d'établir de nouveaux champs de réflexion communs. Concrètement, la mise en place de ce projet nécessite également l'adaptation de la base "Archéomatériaux » aux exigences d'un traitement de ces données par un logiciel de SIG et à sa mise en ligne sur le Web. Outre l'activité nécessaire des opérateurs de données (archéologues, géologues, historiens), l'élaboration de cette interface impose également l'intervention de personnel qualifié en géomatique - pôle géomatique et cartographie de la MSH de Dijon - et de programmateurs qualifiés à la conception et à la mise en ligne de cette base de données de recherche (BRGM).

\section{Le développement du projet}

La mise en place du projet et son suivi ont été placés sous l'égide d'un comité scientifique. Un cahier des charges a été défini dans ce cadre.

\section{Traitement de la base de données « Archéomatériaux »}

La base "Archéomatériaux» nécessite une mise en conformité indispensable au traitement des données par le logiciel de SIG et pour assurer l'interactivité avec les bases partenaires. Ce travail sera réalisé sous la tutelle du BRGM, dans le cadre d'une convention avec l'université de Bourgogne, fort de l'expérience que cette structure possède en ce domaine. Il apparaît également essentiel d'augmenter le corpus, puisque la base, dans son état actuel, ne contient que les données d'études ciblées. La compilation de ce thésaurus est à développer. Cette tâche incombera au Centre d'études médiévales Saint-Germain d'Auxerre, sous le contrôle scientifique du LRMH. 


\section{Traitement SIG} système d'informations géographiques permettra de tester et de développer des modèles d'analyses spatiales, tout en intégrant des facteurs environnementaux, historiques, topographiques, géologiques et techniques. Cette opération sera réalisée en partenariat avec le pôle « Géomatique et cartographique » de la MSH de l'université de Bourgogne.

$\mathrm{Au}$ préalable, une modélisation des données est nécessaire; elle sera réalisée parallèlement à la compilation des données, afin que chaque équipe puisse travailler simultanément - archéologues, géologues, géomaticiens et informaticiens.

\section{Établissement d'un atlas : publication et plateforme Web}

18 À terme, une publication compilant les principaux résultats est envisagée. La démarche vise également à créer un système pérenne, qui pourra continuer à être alimenté au fur et à mesure de l'acquisition de nouvelles données scientifiques: fouilles archéologiques, études du bâti à la faveur de restaurations d'édifices, etc. De fait, le développement d'une plateforme web de consultation permettra d'offrir un véritable outil d'analyse évolutif et à long terme. La compétence du BRGM dans ce domaine sera d'une grande utilité.

\section{NOTES}

1. David Dessandier (BRGM, Service géologique régional Bourgogne Franche-Comté, Dijon); Marion Foucher et Jean-Pierre Garcia (UMR 5594 Artehis, Dijon) ; Lise Leroux (LRMH, Champssur-Marne) ; Christophe Petit (UMR 7041 Arscan, université Paris 1 Panthéon-Sorbonne) ; Laure Saligny (MSH de Dijon, UMS CNRS 2739 Pôle d'économie et de gestion, Dijon) ; Stéphane Büttner et Christian Sapin (Centre d'études médiévales, UMR 5594 Artehis, Auxerre/Dijon) et Jérôme Thomas (UMR 5561 Laboratoire géosciences, Dijon).

2. S. BÜTTNER, « Matériaux et constructions. Sciences et techniques des matériaux, de la carrière à la mise en œuvre : bilan et perspectives »,Bucema,12 (2008), p. 161-168 [http://cem.revues.org/ index6722.html].

3. S. BÜTTNER, "Production et utilisation des pierres bourguignonnes de l'Antiquité à l'époque moderne. L'intérêt d'une base de données ", in R. CARVAIS, A. GUILLERME, V. NÈGRE, et J. SAKAROVITCH, (dir.), Édifice et artifice. Histoire constructive, Paris, 2010, p. 513-522.

4. [http://pierbourgogne.brgm.fr/].

5. [http://www.limestonesculptureanalysis.com/default.asp]. 
INDEX

Index géographique : France/Bourgogne

Mots-clés : atlas, pierre à bâtir, plateforme numérique 